\title{
Self-Assembly Iron Oxide Nanoclusters for Photothermal- Mediated Synergistic Chemo/Chemodynamic Therapy
}

\author{
Xiang Li $\mathbb{D}^{1,2}$ Zhen Wang, ${ }^{3}$ Mian Ma, ${ }^{4}$ Zhouqing Chen $\mathbb{D}^{1},{ }^{1}$ Xiang-long Tang $\mathbb{D},{ }^{3}$ \\ and Zhong Wang ${ }^{1}{ }^{1}$ \\ ${ }^{1}$ Department of Neurosurgery \& Brain and Nerve Research Laboratory, The First Affiliated Hospital of Soochow University, Suzhou, \\ Jiangsu Province, China \\ ${ }^{2}$ Department of Neurosurgery, The Affiliated Hospital of Xuzhou Medical University, Xuzhou, Jiangsu Province, China \\ ${ }^{3}$ Department of Neurosurgery, The Affiliated Brain Hospital with Nanjing Medical University, Fourth Clinical College of Nanjing \\ Medical University, Jiangsu Province, China \\ ${ }^{4}$ Department of Neurosurgery, Suzhou Municipal Hospital, Suzhou, Jiangsu Province, China
}

Correspondence should be addressed to Xiang-long Tang; xltang0326@163.com and Zhong Wang; wangzhong761@163.com

Received 8 March 2021; Revised 24 March 2021; Accepted 27 March 2021; Published 7 April 2021

Academic Editor: Zhi Gang Zhang

Copyright (C) 2021 Xiang Li et al. This is an open access article distributed under the Creative Commons Attribution License, which permits unrestricted use, distribution, and reproduction in any medium, provided the original work is properly cited.

\begin{abstract}
Background and Purpose. Although chemodynamic therapy (CDT) is promising for cancer treatment, its clinical application is still limited because of unresolved issues. In this study, an efficient CDT agent for synergistic chemo/CDT therapy mediated by the photothermal effect was developed by an iron oxide self-assembly method. Methods. Superparamagnetic iron oxide nanoclusters (SPIOCs) were located within the core, which resulted in high photothermal conversion and outstanding generation of reactive oxygen species (ROS). The shell consisted of a human serum albumin- (HSA-) paclitaxel (PTX) layer, which extended the blood circulation time and ensured the effectiveness of the chemotherapy. Arg-Gly-Asp peptides (RGD) were linked to the naked cysteine moieties in HSA to promote the specific targeting of human glioma U87 cells by $\alpha_{\mathrm{v}} \beta_{3}$ integrins. Continuous nearinfrared light irradiation triggered and promoted the synergistic chemo/CDT therapy through the photothermal effect. Results. Our SPIOCs@HSA-RGD nanoplatform showed well biocompatibility and could target glioma specifically. Photothermal conversion and ROS burst were detected after continuous $808 \mathrm{~nm}$ light irradiation, and a significant antitumor effect was achieved. Conclusion. Experimental in vitro and in vivo evaluations showed that our photothermal-mediated chemo/CDT therapy could efficiently inhibit tumor growth and is therefore promising for cancer therapy.
\end{abstract}

\section{Introduction}

In recent decades, conventional therapies for glioma have mainly comprised surgery, chemotherapy, and radiotherapy; however, their therapeutic effect has not been satisfying [ 1 , 2]. Traditional chemotherapy suffers from poor specificity and multiple drug resistance (MDR) and shows substantial toxicity to normal tissues [3-6]. Specific targeting capacity to tumor sites and effective antitumor outcomes are urgently required. Nowadays, nanotechnology-based therapies have presented many promising advantages in this area [7]. Considering the leakage of blood-brain barrier (BBB) and the enhanced permeability and retention (EPR) effect in glioma
$[8,9]$, nanoagents offer unparalleled advantages of penetration and retention in tumor tissue [10], which is of great significance to glioma treatment.

Among various nanocarriers, iron oxide nanoparticles $\left(\mathrm{Fe}_{3} \mathrm{O}_{4} \mathrm{NPs}\right)$ show superior properties, such as low toxicity and excellent biocompatibility and biodegradability, as well as superior photothermal effects [11-14]. Ferumoxytol, an iron oxide-based MRI $\mathrm{T}_{2}$ contrast agent approved by the U.S. Food and Drug Administration (FDA), has been reported [15]. It has been found that some characteristics of $\mathrm{Fe}_{3} \mathrm{O}_{4} \mathrm{NPs}$ are enhanced in ultrasmall superparamagnetic iron oxide nanoclusters (SPIOCs) [16-18]. Once undergoing endocytosis by cancer cells, $\mathrm{Fe}_{3} \mathrm{O}_{4} \mathrm{NPs}$ could function as an 
effective catalyst of Fenton reaction to induce cancer cell death. In addition, the properties of easy preparation, low cost, and controllable size increase the prospects for clinical applications [19]. Meanwhile, under consideration of naked $\mathrm{Fe}_{3} \mathrm{O}_{4}$ NPs which are not fully biocompatible, human serum albumin (HSA), with its inherent biocompatibility and abundance, is usually used to decrease their toxicity [20]. These HSA-coated nanoparticles can be internalized into cancer cells by gp60 receptor-related translation mechanism that overcomes negative charge-induced repulsion. In addition, Arg-Gly-Asp (RGD) peptide, a classical binding motif targeting $\alpha_{\mathrm{v}} \beta_{3}$ integrins of tumors, could enhance the cancer binding affinity and cellular uptake of nanoparticles [21]. Thus, a nanosystem could specifically accumulate in glioma tissues by enhanced permeable reaction (EPR) as well as the assistance of RGD peptide [22].

Photothermal therapy is a noninvasive and controllable strategy superior to other traditional therapies [23]. Since any single therapy still suffers from limited efficacy, photothermal combination therapies are used to increase the efficacy of chemotherapeutic drugs for destroying tumor tissues at the same dose, and they are commonly combined with other therapies, such as chemotherapy and chemodynamic therapy (CDT) $[24,25]$. Although reactive oxygen species (ROS) mediate physiological and pathophysiological signal transduction, excessive cytotoxic ROS, which cause oxidative stress, lipid peroxidation, and DNA damage in tumors [26,27], have recently been used to treat glioma [28]. Exogenous ROS, such as hydroxyl radicals $(\mathrm{OH})$ and superoxide anions $\left(\mathrm{O}_{2}{ }^{-}\right)$are toxic and have high oxidation capability [29]. In the presence of iron oxide NPs, large amounts of ROS are produced by the Fenton reaction, which converts hydrogen peroxide $\left(\mathrm{H}_{2} \mathrm{O}_{2}\right)$ into cytotoxic hydroxyl radicals $(\mathrm{OH})$; this reaction is promoted by the higher local temperature generated by the photothermal effect [30-32]. Excessive ROS could not only promote chemotherapy but also stimulate drug release from nanocarriers, which is of great significance in tumor chemotherapy $[33,34]$.

In our work, we aimed to promote the synergy efficiency of chemotherapy and chemodynamic therapy by means of ROS burst from the Fenton reaction mediated by photothermal conversion (Figure 1). We designed a novel nanoplatform (SPIOCs@HSA(PTX)-RGD) with superparamagnetic $\mathrm{Fe}_{3} \mathrm{O}_{4}$ nanoclusters (SPIOCs) as the spheroid core coated with a HSA-PTX layer. The SPIOCs@HSA(PTX)-RGD nanoplatform showed good biocompatibility and excellent specific targeting, which was attributed to the HSA and RGD ligands. It could be internalized in tumor cells via endocytosis and accumulated in sufficient concentrations through enhanced permeable reaction (EPR). Irradiation with nearinfrared (NIR) laser light $(808 \mathrm{~nm})$ resulted in enhanced release of drugs. Further, the catalytic SPIOCs and SPIOCinduced photothermal conversion promoted the ironmediated Fenton reaction to generate hydroxyl radicals and enhance the ROS level, which triggered tumor apoptosis and ablation. Photothermal-mediated chemo/chemodynamic therapy could inhibit tumor growth more efficiently than any single therapy. The antitumor efficiency of the SPIOCs@HSA(PTX)-RGD nanoplatform was demonstrated by both in vivo and in vitro experiments.

\section{Material and Methods}

2.1. Materials. Iron(III) acetylacetonate $\left(\mathrm{Fe}(\mathrm{acac})_{3}\right)$, oleic acid, oleylamine, and ethanol were purchased from Aladdin (China). DMEM, fetal bovine serum (FBS), and penicillinstreptomycin were purchased from Gibco (USA). HSA was purchased from Beijing Biosynthesis Biotechnology Co., Ltd. Thiolated RGD peptides were obtained from China Peptides Co., Ltd. Sulfo-SMCC, 4,6-diamidino-2-phenylindole (DAPI), and DCFH-DA probe were purchased from Sigma-Aldrich (USA). TUNEL apoptosis assay kit was obtained from Beyotime Biotechnology (China).

\subsection{Preparation of Ultrasmall Superparamagnetic Iron Oxide} Nanoparticles. Ultrasmall superparamagnetic iron oxide nanoparticles were synthesized by the procedure reported in a previous study [35]. In brief, equal volumes of oleic acid and oleylamine $(15 \mathrm{~mL}), \mathrm{Fe}(\mathrm{acac})_{3}(9 \mathrm{mmol})$ and benzyl ether $(40 \mathrm{~mL})$ were mixed and homogenized by mild mechanical stirring. The temperature was continuously heated to $210^{\circ} \mathrm{C}$, and the solution was refluxed at this temperature for 6 hours under a nitrogen atmosphere. Superparamagnetic iron oxide NPs were synthesized when the solution color changed from brown to black and were precipitated with excess ethanol. Then, superparamagnetic iron oxide NPs were separated by centrifugation. After washing with absolute ethanol, the particles were resuspended in chloroform.

2.3. Synthesis of SPIOCs@HSA(PTX) NPs. SPIOCs@HSA(PTX) NPs were synthesized by the following steps: monodispersed iron oxide nanoparticles were dissolved in chloroform and then mixed with HSA solution ( $\mathrm{pH} 7.4$, $26.5 \mathrm{mg} / \mathrm{mL}$ ). Ultrasound vibration was carried out at $5 \mathrm{~min}$ intervals to prevent the generation of high temperatures. After evaporation of the organic chloroform phase by a rotary evaporator, a diaphanous SPIOCs@HSA solution was obtained. PTX was dissolved in DMSO and added dropwise to the SPIOCs@HSA solution under ultrasonic vibration for at least 5 min. SPIOCs@HSA(PTX) NPs were obtained after dialysis with a membrane (MW: $8 \mathrm{kDa})$.

2.4. Synthesis of SPIOCs@HSA(PTX)-RGD NPs. Thiolated RGD peptide was conjugated to enhance the tumor-targeting specificity with a method similar to that described in our previous report [36]. In brief, SPIOCs@HSA(PTX) solution (pH 7.4) was incubated with Sulfo-SMCC dissolved in DMSO. The solution was placed in an oscillating shaker $(130 \mathrm{rpm})$ for half an hour at $25^{\circ} \mathrm{C}$. Subsequently, maleimide-decorated SPIOCs@HSA(PTX)-Mal were centrifuged (16000g), and thiolated RGD peptide was added to the SPIOCs@HSA(PTX)-Mal solution (pH 7.4) at $4^{\circ} \mathrm{C}$ overnight. After centrifugation in filtration tubes $\quad(\mathrm{MWCO}=8000-12000 \mathrm{Da}), \quad$ purified SPIOCs@HSA(PTX)-RGD NPs were obtained.

2.5. Characterization of the Nanoparticles. The morphology was characterized by transmission electron microscopy (TEM, JEOL JEM-2100). The hydrodynamic radii and zeta potentials of the nanoparticles were measured by a laser granularity analyzer (Malvern Zetasizer, UK). 


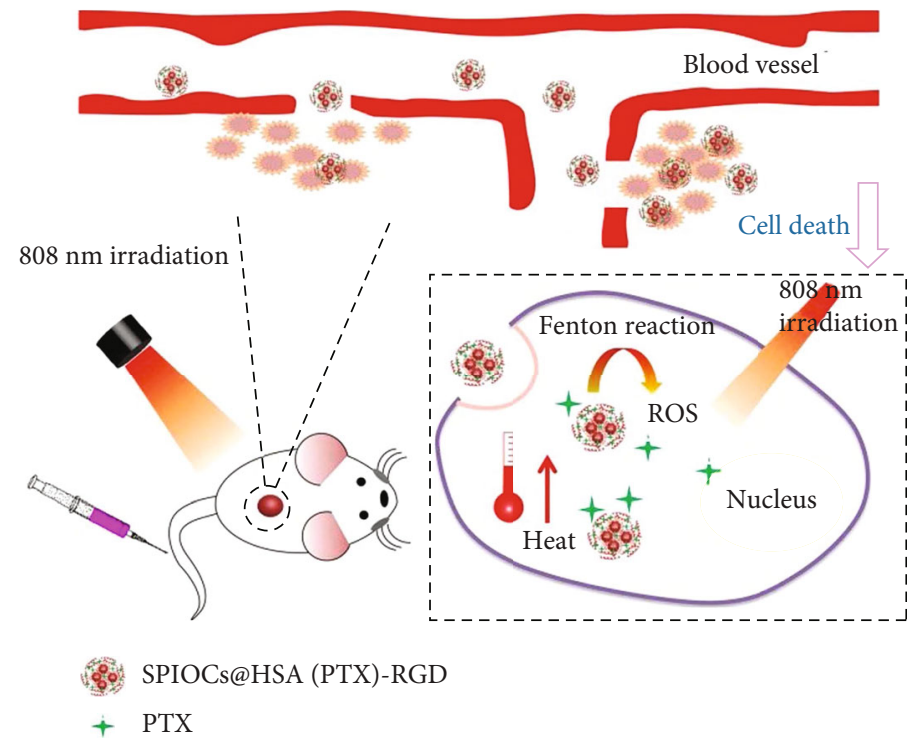

FIGURE 1: Schematic illustration of SPIOCs@HSA(PTX)-RGD nanoplatforms applied in photothermal mediated chemo/chemodynamic therapy.

2.6. Photothermal Conversion Efficiency In Vitro. An aqueous solution of SPIOCs@HSA(PTX)-RGD NPs $(60 \mu \mathrm{g} \mathrm{Fe} / \mathrm{mL})$ was irradiated by $808 \mathrm{~nm}$ near-infrared laser $\left(0.5 \mathrm{~W} / \mathrm{cm}^{2}\right)$ to evaluate the photothermal properties. An infrared thermal imager (Fluke TiS20) equipped with a temperature-sensitive probe was used to monitor the real-time thermal behaviors per second during irradiation.

2.7. Cellular Uptake. Human glioma U87 cells in logarithmic growth were seeded in confocal laser petri dishes (diameter: $35 \mathrm{~mm}$ ) at the density of $10^{5} /$ well and cultured in DMEM with 10\% FBS for 24 hours (saturated humidity, 5\% carbon dioxide, $\left.37^{\circ} \mathrm{C}\right)$. Then, the U87 cells were incubated with Ce6-labeled SPIOCs@HSA-RGD NPs for 4 hours. Afterwards, they were fixed with $4 \%$ paraformaldehyde, and their nuclei were labeled with DAPI. The U87 cells were washed with PBS solution ( $\mathrm{pH} 7.4$ ) several times, and the fluorescence signals were observed by confocal laser scanning microscopy (CLSM).

2.8. Cell Viability Assays. The cell cytotoxicity was measured by the MTT assay. Human glioma U87 cells were seeded and cultured in 96-well plates (DMEM medium with 10\% FBS and 1\% penicillin-streptomycin). The SPIOCs@HSA(PTX), SPIOCs@HSA(PTX)-RGD, and SPIOCs@HSA(PTX)-RGD +irradiated groups with different PTX concentrations were incubated with the U87 cells, and PBS was used as the control. After $12 \mathrm{~h}$ of incubation, the SPIOCs@HSA(PTX)RGD+irradiated group was exposed to $808 \mathrm{~nm}$ NIR laser illumination $\left(0.5 \mathrm{~W} / \mathrm{cm}^{2}\right)$ for half an hour after the culture medium was replaced by PBS. Then, the cells were incubated with fresh culture medium for $24 \mathrm{~h}$. Finally, the MTT assay was carried out. To prevent drugs from reacting with MTT, the original culture medium was discarded, and MTT-containing culture medium (serum-free medium : MTT $=5: 1$ ) was added. After $4 \mathrm{~h}$ of culture in the dark, the medium was discarded, and DMSO was added. All wells were mixed evenly by placing the plate on a shaker, and the absorbance was measured with a microplate reader.

2.9. Animal Model. Male Balb/c nude rats (4-5 weeks) were purchased from Nanjing Biomedical Research Institute of Nanjing University, and subcutaneous xenograft models were established; all experiments were carried out under the permission and guidelines of the Ethics Committee of Nanjing Medical University. The U87 cells were collected by resuspension and centrifugation in serum-free medium, and $3 \times 10^{6}$ cells were injected into the right flank region of each rat subcutaneously. The rats were housed in an SPFgrade environment.

2.10. In Vivo Fluorescence Imaging and Biodistribution. Two hundred microliters of HSA(PTX), SPIOCs@HSA(PTX), and SPIOCs@HSA(PTX)-RGD was injected into randomly selected tumor-bearing nude rats via the tail vein (labeled with Ce6, Ce6: $1.90 \mathrm{mg} / \mathrm{kg}$ ). After administration of anesthesia with isoflurane, in vivo biodistributions were detected by IVIS spectrum small-animal imaging system (PerkinElmer, USA). The rats were euthanized under thoroughly anesthetizing with isofluorane 10-hour postinjection. Tumors and normal organs (heart liver, lungs, kidneys, and spleen) were excised immediately for ex vivo bioluminescence imaging. The excised tumors were fixed with $10 \%$ neutral formalin tissue fixative. Prussian blue-eosin double staining was performed on $5 \mathrm{~mm}$ paraffin slices by experienced pathologists with blind samples.

2.11. In Vivo Photothermal Conversion. SPIOCs@HSA(PTX)-RGD NPs were injected into tumor-bearing rats. Under isofluorane anesthesia, the tumor sites were exposed to $808 \mathrm{~nm}$ laser irradiation $\left(0.5 \mathrm{~W} / \mathrm{cm}^{2}\right) 10$-hour postinjection. The temperature changes were recorded by an infrared thermal imager. 


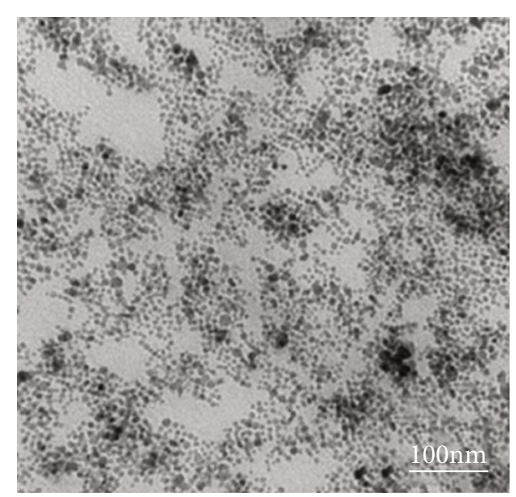

(a)

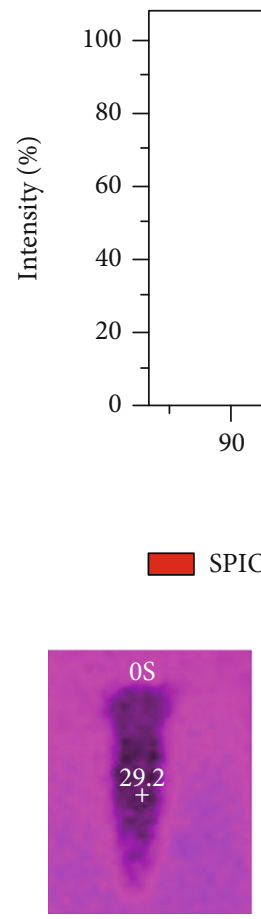

SPIOCs@HSA (PTX)-RGD

(c)

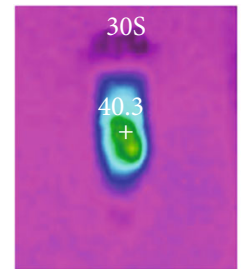

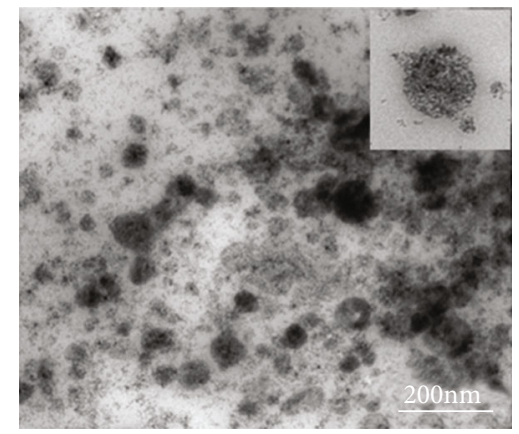

(b)

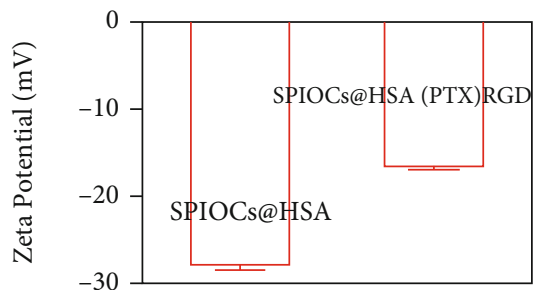

(d)
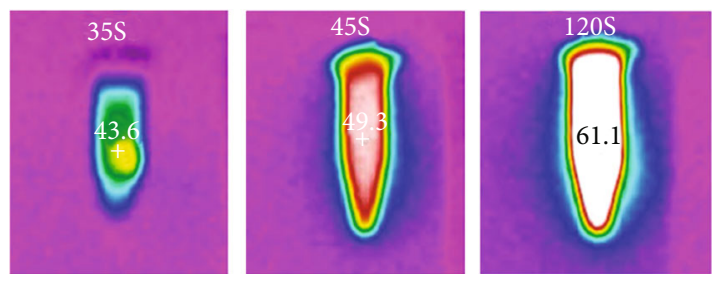

(e)

FIgure 2: TEM morphologies of (a) ultrasmall superparamagnetic $\mathrm{Fe}_{3} \mathrm{O}_{4}$ NPs and (b) SPIOCs@HSA(PTX)-RGD; (c) Size distribution determined by DLS; (d) zeta potential of SPIOCs@HSA and SPIOCs@HSA(PTX)-RGD NPs; (e) photothermal temperatures of the SPIOCs@HSA(PTX)-RGD irradiated by $808 \mathrm{~nm}$ light $\left(0.5 \mathrm{~W} / \mathrm{cm}^{2}\right)$.

2.12. In Vivo Antitumor Efficiency. U87 glioma subcutaneous xenograft models were randomly divided into 4 groups $(n=5$ ) , and different treatments were administered by tail vein intravenous injection. The groups were (1) PBS, (2) SPIOCs@HSA(PTX), (3) SPIOCs@HSA(PTX)-RGD, and (4) SPIOCs@HSA(PTX)-RGD+808 nm laser irradiation at $10 \mathrm{~h}$ postinjection. The tumor volume (Equation (1)) was recorded every two days with a vernier caliper.

Tumor volume $(V)=\left(\right.$ major axes $\times$ minor axes $\left.{ }^{2}\right) \times 0.5$,

Relative value $\left(V_{\mathrm{r}}\right)=V / V_{0}\left(V_{0}\right.$ : original tumor volume $)$.

Equation (2) was used to calculate the relative tumor vol- ume changes. The treatments began when the tumor size reached approximately $100 \mathrm{~mm}^{3}$, and the rats were sacrificed after 14 days. The tumors were dissected for weight measurement and further study.

2.13. In Vivo ROS and Apoptosis Detection. To analyze the ROS accumulation, frozen tumor sections from each group at 4-day posttreatment were incubated with the ROSsensitive probe dihydroethidium (DHE) for $30 \mathrm{~min}$. The nuclei were labeled with DAPI. The fluorescence was detected by fluorescence microscopy.

To detect the apoptosis induced by the ROS burst, a TUNEL apoptotic fluorescence assay was performed. In brief, frozen slices taken at day 4 after different treatments were fixed with $4 \%$ formalin, blocked with $3 \% \mathrm{H}_{2} \mathrm{O}_{2}$, and immersed in permeable solution. The slices were incubated 

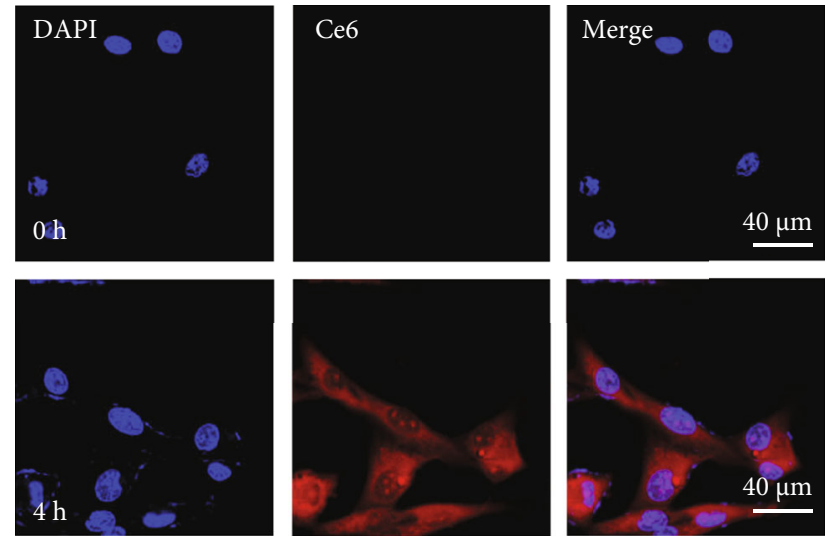

(a)

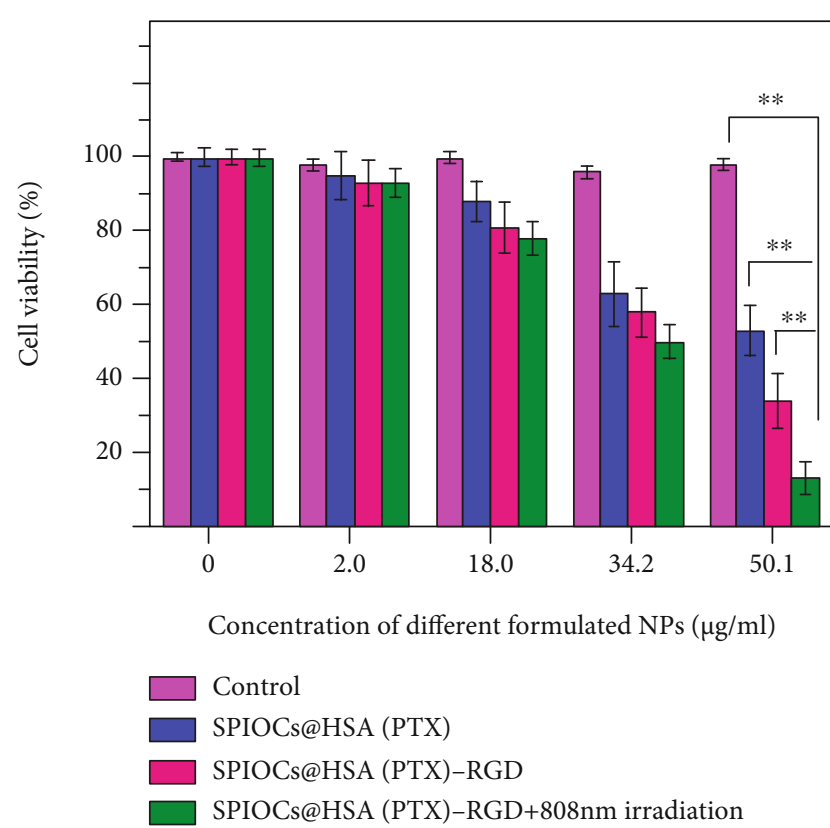

(b)

FIGURE 3: (a) Internalization of Ce6-labeled SPIOCs@HSA-RGD into U87 cells after incubation for 4 h; (b) in vitro antitumor ability of SPIOCs@HSA(PTX)-RGD at different PTX concentrations $\left({ }^{* *} P<0.01\right)$.

with the TUNEL solution containing TDT enzyme and fluorescent labeling solution for $1 \mathrm{~h}$ in the dark. After washing the slices with PBS three times, the nuclei were labeled with DAPI. Apoptosis was detected via the stains by fluorescence microscopy. The fluorescence staining experiments above were all conducted and analyzed by experienced pathologists with blind samples.

2.14. Statistics. GraphPad Prism 7.0 was used for statistical analysis. Student's $t$-test was utilized to assess differences between distinct groups. $P$ values less than 0.05 were supposed to be statistically significant. Experimental results were presented as the mean \pm standard deviation.

\section{Results and Discussion}

3.1. Synthesis and Characterization. A solvothermal synthesis protocol was applied to prepare ultrasmall $\mathrm{Fe}_{3} \mathrm{O}_{4}$ nanoparticles. Hydrophobic ultrasmall superparamagnetic iron oxide nanoclusters (SPIOCs), which were employed as the core of our SPIOCs@HSA(PTX)-RGD nanoplatform, were shelled by the hydrophilic human serum albumin (HSA). The SPIOCs played an important role in chemodynamic therapy and photothermal conversion. HSA was decorated on SPIOCs for long-term blood circulation via a self-assembly method. PTX, which could inhibit mitosis and trigger cancer cell apoptosis, was used as the shell and coated onto the surface of the SPIOCs via hydrophobic interactions with HSA. To increase tumor specificity, RGD peptide was conjugated onto the SPIOCs@HSA(PTX) NPs for targeting the abundant $\alpha_{\mathrm{v}} \beta_{3}$-integrin on the glioma cells.

The morphology and structure were identified by transmission electron microscopy (TEM). Images of the ultra- small superparamagnetic $\mathrm{Fe}_{3} \mathrm{O}_{4}$ NPs (Figure 2(a)) showed a relatively uniform spherical morphology of which the diameter was less than $10 \mathrm{~nm}$. In order to obtain monodispersed SPIOCs@HSA(PTX)-RGD nanoclusters, an ultrasound vibration-assisted self-assembly technique had been applied in our preparation. The morphology of SPIOCs@HSA(PTX)-RGD nanoclusters was revealed by TEM images (Figure 2(b)), which were uniformly dispersed with an average diameter $\approx 95 \mathrm{~nm}$. The typical hydrodynamic radius of the SPIOCs@HSA(PTX)-RGD (112 $\pm 10.5 \mathrm{~nm})$ was obtained via dynamic light scattering (DLS) (Figure 2(c)). The zeta potential (surface charge) of the SPIOCs@HSA(PTX)-RGD NPs $(\sim-16 \mathrm{mV})$ was higher than that of the SPIOCs@HSA NPs $(\sim-28 \mathrm{mV})$ (Figure 2(d)), indicating a more stable structure and less resistance for the internalization in glioma cells.

3.2. In Vitro Photothermal Conversion. Near-infrared (NIR) laser light can penetrate biological tissues more efficiently and inflicts less damage than ultraviolet light. The excellent photothermal conversion effect of our SPIOCs@HSA(PTX)-RGD NPs was mainly attributed to the SPIOCs core. When irradiated with $808 \mathrm{~nm}$ NIR laser light $\left(0.5 \mathrm{~W} / \mathrm{cm}^{2}\right)$, the temperature of SPIOCs@HSA(PTX)-RGD NPs solution increased rapidly by nearly 20 degrees during the initial $45 \mathrm{~s}$ and continued to increase slowly over the subsequent period (Figure 2(e)). Within $120 \mathrm{~s}$, the temperature increased by more than $30^{\circ} \mathrm{C}$ and nearly reached $61.1^{\circ} \mathrm{C}$, which resulted in the sufficient destruction of tumor cells.

3.3. In Vitro Endocytosis and Cytotoxicity. To evaluate the capacity of the SPIOCs@HSA-RGD NPs to target human glioma U87 cells overexpressing $\alpha_{\mathrm{v}} \beta_{3}$ integrin, an uptake study was performed, and the internalization was investigated 
(a)
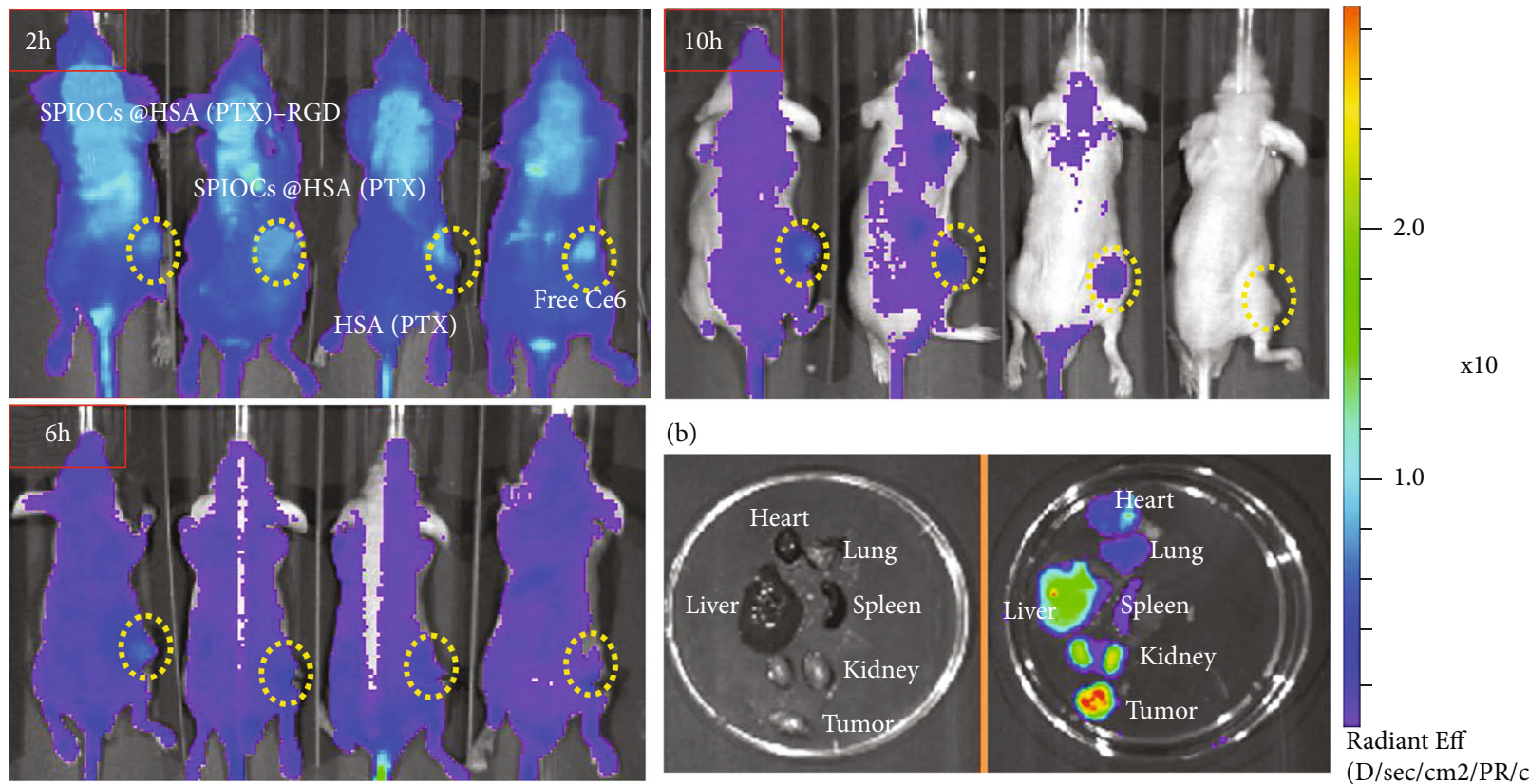

(b)
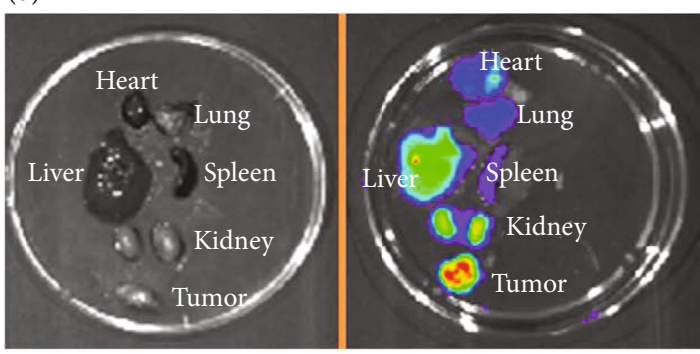

$(\mathrm{D} / \mathrm{sec} / \mathrm{cm} 2 / \mathrm{PR} / \mathrm{c}$

(c)

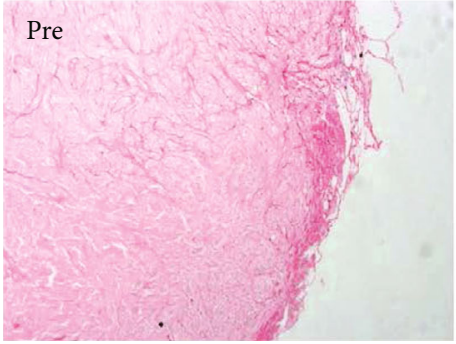

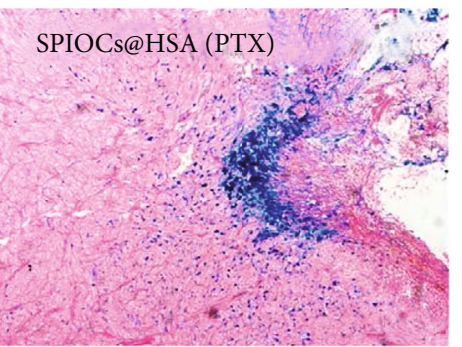

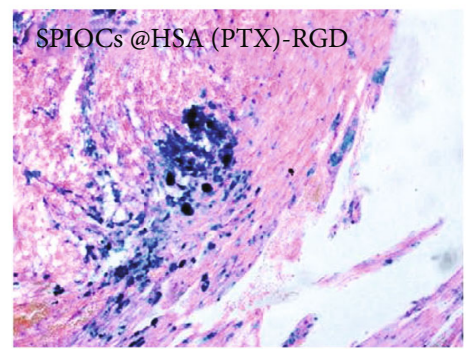

FIGURE 4: (a) Biodistribution of different nanoparticle groups recorded by IVIS fluorescence images at 2-hour, 6-hour, and 10-hour postinjection. (b) Bioluminescence signals of ex vivo tumor and normal organs including the heart, lung, liver, spleen, and kidneys of SPIOCs@HSA(PTX)-RGD group. (c) Prussian blue-eosin double staining of tumor sections. Prussian blue-stained regions represented the iron nanoparticles deposited in tumor beds 10-hour postinjection.

by confocal microscopy. The SPIOCs@HSA-RGD NPs were labeled with fluorescent Chlorin e6 (Ce6). After incubation with the U87 cells for 4 hours, strong Ce6 red fluorescence signals were observed both in the cytoplasm and nucleus (Figure 3(a)). This endocytosis might be attributed to the HSA and RGD peptide on the particles interacting with the albumin gp60 receptor and $\alpha_{\mathrm{v}} \beta_{3}$ integrins overexpressed on the surface of glioma cells.

Moreover, the biocompatibility and cytotoxicity of this nanoplatform were evaluated by a cell viability assay (Figure 3(b)). SPIOCs@HSA(PTX)-RGD did not show significant cytotoxicity towards U87 cells when the concentration of PTX was below $18 \mu \mathrm{g} / \mathrm{mL}$. The inhibitory effect of the different NP groups on the U87 cells was evaluated. Interestingly, SPIOCs@HSA(PTX)-RGD was much cytotoxic than those without RGD peptide decoration at a PTX concentration of $34.2 \mu \mathrm{g} / \mathrm{mL}$; further, the similar phenomenon was observed when the PTX concentration exceeded $50 \mu \mathrm{g} / \mathrm{mL}$. And this phenomenon was attributed to the RGD targeting capacity to U87 cells, which was beneficial for tumor inhibition. The synergistic SPIOCs@HSA(PTX)-RGD $+808 \mathrm{~nm}$ irradiated group resulted in the best antitumor effect among all the groups, which demonstrated the superiority of photothermally mediated chemo/chemodynamic therapy.
3.4. In Vivo Dynamic Biodistribution. To investigate the accumulation and distribution of the SPIOCs@HSA(PTX)RGD NPs in the tumor environment, Ce6-labeled NPs were intravenously injected into U87-bearing mice, and their in vivo biodistribution was evaluated at different time points (Figure 4(a)). Over time, the fluorescence signal intensity at the tumor site increased, whereas it gradually decreased at other sites. Six-hour postinjection, the aggregation of the SPIOCs@HSA(PTX)-RGD in the tumor environment was more pronounced than that of SPIOCs@HSA(PTX), HSA(PTX), and free Ce6 groups, indicating good targeting capability. After 10 hours, free Ce6 was completely biocleared, while a large amount of SPIOCs@HSA(PTX)RGD NPs were retained in the blood circulation, showing the longest half-value period among the different groups. The fluorescence biodistribution in the tumors and major organs at 10-hour postinjection also confirmed the specific targeting capability of our SPIOCs@HSA(PTX)-RGD NPs (Figure 4(b)). Furthermore, Prussian blue/eosin staining of tumor specimen slices (Figure 4(c)) indicated the iron distribution (blue region) in the tumor tissue 10 hours after injection. The SPIOCs@HSA(PTX)-RGD group showed the maximum density of iron compared with the other groups, indicating that iron oxide nanoparticles penetrated into the 


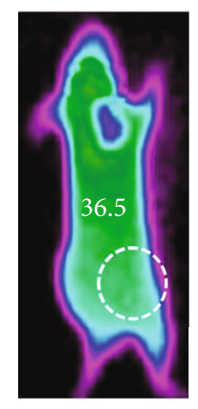

$0 \mathrm{~s}$

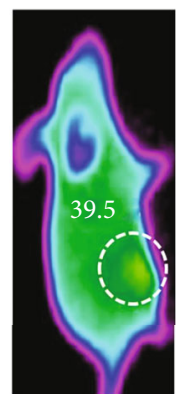

$10 \mathrm{~s}$

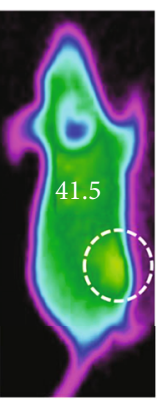

$20 \mathrm{~s}$

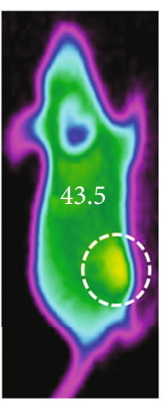

$40 \mathrm{~s}$
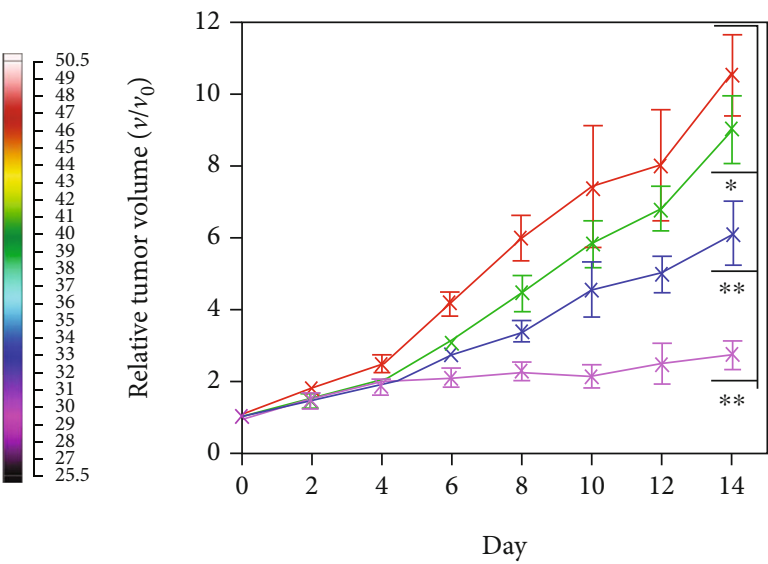

$*$ PBS

* SPIOCs@HSA (PTX)-RGD

$\rightarrow$ SPIOCs@HSA (PTX)-RGD+808nm

$*$ SPIOCs@HSA (PTX)

(b)
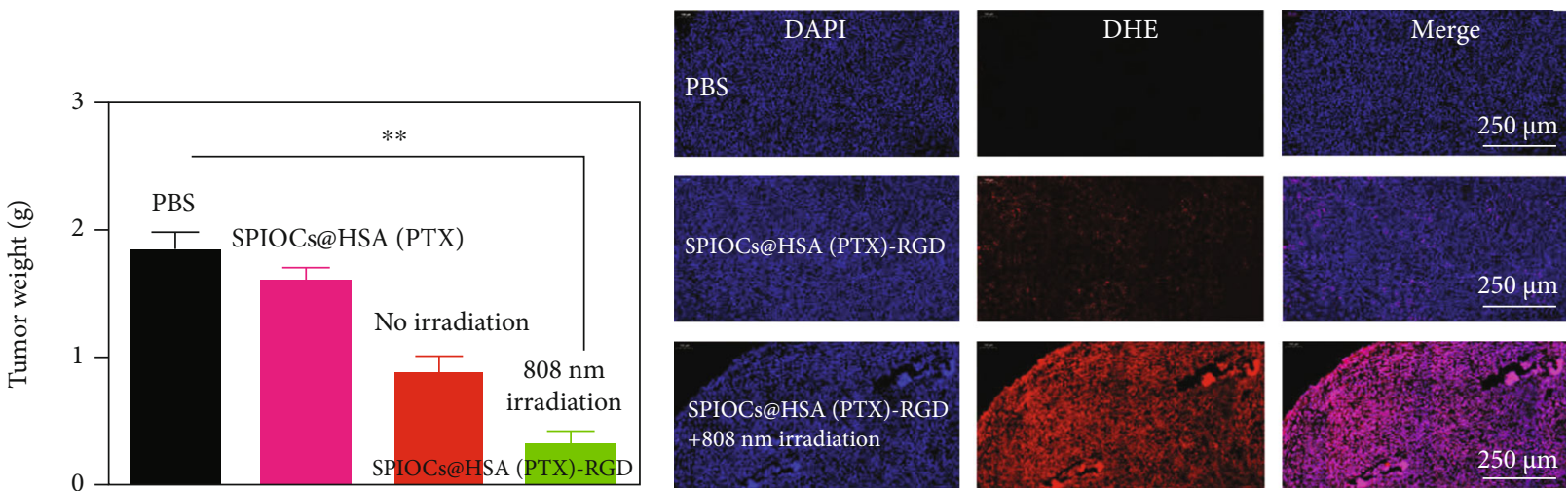

(c)
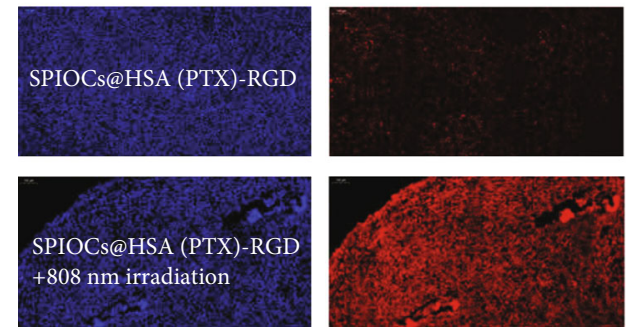

(d)

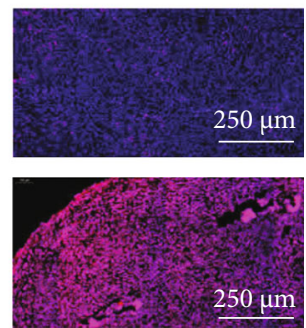

Figure 5: Continued. 

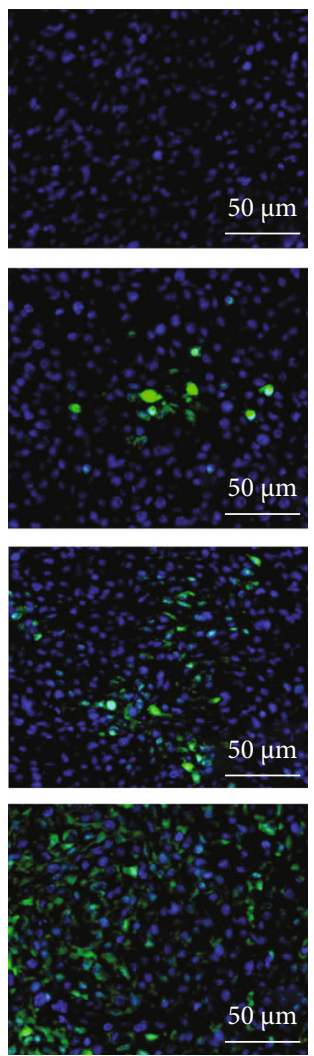

(e)

Figure 5: (a) Real-time infrared thermal images of tumor-bearing rats exposed to laser irradiation $\left(808 \mathrm{~nm}, 0.5 \mathrm{~W} / \mathrm{cm}^{2}\right) 10 \mathrm{hours}$ after the injection of SPIOCs@HSA(PTX)-RGD NPs; (b) in vivo tumor growth curves of the glioma-bearing mice after the different treatments $\left({ }^{*} P<0.05\right.$, $\left.{ }^{* *} P<0.01\right)$; (c) tumor weights measured after the various treatments $\left({ }^{* *} P<0.01\right)$; (d) oxidative stress detected by ROS probe; (e) TUNEL-stained tumor slices taken at day 4; groups from top to bottom are PBS, SPIOCs@HSA(PTX), SPIOCs/@HSA(PTX)-RGD, and SPIOCs@HSA(PTX)-RGD+808 nm irradiation, respectively.

interior of the tumor tissues effectively, which was consistent with the fluorescence results.

3.5. In Vivo Synergistic Therapy for Glioma. The photothermal effect of SPIOCs@HSA(PTX)-RGD NPs in vivo was verified by full-body infrared thermography of nude rats bearing U87 glioma. After intravenous injection of the SPIOCs@HSA(PTX)-RGD NPs, hyperthermia at the tumor site was observed in real time when irradiated with NIR light $\left(808 \mathrm{~nm}, 0.5 \mathrm{~W} / \mathrm{cm}^{2}\right)$ (Figure 5(a)), indicating an excellent photothermal conversion in vivo.

To further examine the tumor inhibition ability, the corresponding treatments were applied to subcutaneous U87 glioma, and the tumor volumes were recorded to evaluate the respective antitumor effects. The SPIOCs@HSA(PTX)RGD NP group most efficiently inhibited the tumor growth compared with the other groups treated with free PBS solution and SPIOCs@HSA(PTX) NPs. When synergistic therapy of SPIOCs@HSA(PTX)-RGD NPs + NIR light irradiation was applied, the tumor growth was inhibited even more (Figure 5(b)). Compared with groups without NIR irradiation, a substantial difference was observed in the tumor weights after 14 days (Figure 5(c)).
The production of reactive oxygen species (ROS) and oxidative stress were determined by the ROS probe (DHE). Slices of the tumors treated with synergistic therapy of SPIOCs@HSA(PTX)-RGD NPs + NIR light irradiation were remarkably brighter with a more intense red fluorescence than those without irradiation (Figure 5(d)). This result showed that a large amount of ROS, such as superoxide anions and hydroxyl radicals, were produced by the synergistic therapy. These ROS were produced by the SPIOC-catalyzed Fenton reaction at elevated temperature due to photothermal conversion. The ROS generation mediated by photothermal conversion synergistically enhanced the cell apoptosis, which could be visualized by the apoptosis fluorescence of the tumor slices taken at day 4 (Figure 5(e)). All the results indicated that our synergistic therapy is promising for application in vivo.

\section{Conclusion}

In summary, we developed an efficient SPIOCs@HSA(PTX)RGD nanoplatform for photothermal-mediated chemo/chemodynamic therapy. Superparamagnetic iron oxide nanoclusters (SPIOCs) triggered photothermal conversion and efficiently catalyzed the Fenton reaction. Coating with human serum 
albumin (HSA) resulted in high stability and biocompatibility of the nanoplatform. Conjugated RGD peptide ligands allowed for efficient targeting of human U87 glioma. With the assistance of NIR light irradiation, excessive cytotoxic ROS generated from photothermally mediated Fenton reaction coupled with chemotherapy resulted in glioma apoptosis. This combination therapy was more effective in promoting glioma ablation than any single treatment. Considering the glioma growth inhibition, specific targeting ability, and biosafety, our photothermal-mediated synergistic chemo/chemodynamic treatment is promising for use in the clinic.

\section{Data Availability}

The data used to support the findings of this study are included within the article.

\section{Conflicts of Interest}

The authors declare no conflict of interest.

\section{Authors' Contributions}

Xiang Li and Zhen Wang contributed equally to this work.

\section{Acknowledgments}

This work was financially supported by the National Natural Science Foundation of China, grant numbers 81902535 and 81972350; the China Postdoctoral Science Foundation, grant number 2019M651896; the clinical medical special Program of Science and Technology Project of Jiangsu Province, grant number BE2019734; Medical Research Foundation of Jiangsu Health Commission, grant number H2019059; Science and Technology Development Foundation of Nanjing Medical University, grant number NMUB 2018095; and Medical Science and Technology Development Foundation of Nanjing, grant number YKK19088.

\section{References}

[1] S. Lapointe, A. Perry, and N. A. Butowski, "Primary brain tumours in adults," The Lancet, vol. 392, no. 10145, pp. 432446, 2018.

[2] M. Lim, Y. Xia, C. Bettegowda, and M. Weller, "Current state of immunotherapy for glioblastoma," Nature Reviews Clinical oncology, vol. 15, no. 7, pp. 422-442, 2018.

[3] M. Gao, F. Yu, C. Lv, J. Choo, and L. Chen, "Fluorescent chemical probes for accurate tumor diagnosis and targeting therapy," Chemical Society Reviews, vol. 46, no. 8, pp. 2237-2271, 2017.

[4] A. Thomas, M. Tanaka, J. Trepel, W. C. Reinhold, V. N. Rajapakse, and Y. Pommier, "Temozolomide in the era of precision medicine," Cancer Research, vol. 77, no. 4, pp. 823-826, 2017.

[5] M. Ramirez, S. Rajaram, R. J. Steininger et al., "Diverse drugresistance mechanisms can emerge from drug-tolerant cancer persister cells," Nature Communications, vol. 7, no. 1, article 10690, 2016.
[6] D. Uribe, Á. Torres, J. D. Rocha et al., "Multidrug resistance in glioblastoma stem-like cells: role of the hypoxic microenvironment and adenosine signaling," Molecular Aspects of Medicine, vol. 55, pp. 140-151, 2017.

[7] J. S. Michael, B.-S. Lee, M. Zhang, and J. S. Yu, "Nanotechnology for treatment of glioblastoma multiforme," J Transl Int Med., vol. 6, no. 3, pp. 128-133, 2018.

[8] S. Liebner, A. Fischmann, G. Rascher et al., "Claudin-1 and claudin-5 expression and tight junction morphology are altered in blood vessels of human glioblastoma multiforme," Acta Neuropathologica, vol. 100, no. 3, pp. 323-331, 2000.

[9] H. Maeda, "The enhanced permeability and retention (EPR) effect in tumor vasculature: the key role of tumor-selective macromolecular drug targeting," Advances in Enzyme Regulation, vol. 41, no. 1, pp. 189-207, 2001.

[10] B. Delgado-Martín and M. Á. Medina, "Advances in the knowledge of the molecular biology of glioblastoma and its impact in patient diagnosis, stratification, and treatment," Adv Sci (Weinh)., vol. 7, no. 9, p. 1902971, 2020.

[11] N. S. Vallabani and S. Singh, "Recent advances and future prospects of iron oxide nanoparticles in biomedicine and diagnostics," 3 Biotech, vol. 8, no. 6, p. 279, 2018.

[12] S. Shen, S. Wang, R. Zheng et al., "Magnetic nanoparticle clusters for photothermal therapy with near-infrared irradiation," Biomaterials, vol. 39, pp. 67-74, 2015.

[13] F.-Y. Cheng, C.-H. Su, Y.-S. Yang et al., "Characterization of aqueous dispersions of $\mathrm{Fe}$ (3) $\mathrm{O}$ (4) nanoparticles and their biomedical applications," Biomaterials, vol. 26, no. 7, pp. 729-738, 2005.

[14] L.-h. Shen, J.-f. Bao, D. Wang et al., "One-step synthesis of monodisperse, water-soluble ultra-small $\mathrm{Fe} 3 \mathrm{O} 4$ nanoparticles for potential bio-application," Nanoscale, vol. 5, no. 5, pp. 2133-2141, 2013.

[15] S. Zanganeh, G. Hutter, R. Spitler et al., "Iron oxide nanoparticles inhibit tumour growth by inducing pro-inflammatory macrophage polarization in tumour tissues," Nature Nanotechnology, vol. 11, no. 11, pp. 986-994, 2016.

[16] Q. Wang, S. Wang, X. Hu, F. Li, and D. Ling, "Controlled synthesis and assembly of ultra-small nanoclusters for biomedical applications," Biomaterials Science, vol. 7, no. 2, pp. 480-489, 2019.

[17] X. Meng, I. Zare, X. Yan, and K. Fan, "Protein-protected metal nanoclusters: an emerging ultra-small nanozyme," WIREs Nanomedicine and Nanobiotechnology, vol. 12, no. 3, article e1602, 2020.

[18] B. Rajan, S. Sathish, S. Balakumar, and T. Devaki, "Synthesis and dose interval dependent hepatotoxicity evaluation of intravenously administered polyethylene glycol-8000 coated ultra-small superparamagnetic iron oxide nanoparticle on Wistar rats," Environmental Toxicology and Pharmacology, vol. 39, no. 2, pp. 727-735, 2015.

[19] D. H. Huang, X. Y. Qi, Y. R. Ge, and S. Shen, “Application of magnetic iron oxide nanoparticles in magnetic resonance/photothermal dual-modal imaging," Yao xue xue bao = Acta Pharmaceutica Sinica, vol. 52, no. 3, pp. 481-487, 2017.

[20] Q. Chen, X. Wang, C. Wang, L. Feng, Y. Li, and Z. Liu, "Druginduced self-assembly of modified albumins as nanotheranostics for tumor-targeted combination therapy," ACS Nano, vol. 9, no. 5, pp. 5223-5233, 2015.

[21] P.-H. Wu, A. E. Opadele, Y. Onodera, and J.-M. Nam, “Targeting integrins in cancer nanomedicine: applications in cancer diagnosis and therapy," Cancers, vol. 11, no. 11, p. 1783, 2019. 
[22] J. Gong, M. Chen, Y. Zheng, S. Wang, and Y. Wang, "Polymeric micelles drug delivery system in oncology," Journal of Controlled Release, vol. 159, no. 3, pp. 312-323, 2012.

[23] W. Wei, X. Zhang, S. Zhang, G. Wei, and Z. Su, "Biomedical and bioactive engineered nanomaterials for targeted tumor photothermal therapy: a review," Materials Science \& Engineering. C, Materials for Biological Applications, vol. 104, p. 109891, 2019.

[24] M. Wu, Y. Ding, and L. Li, "Recent progress in the augmentation of reactive species with nanoplatforms for cancer therapy," Nanoscale, vol. 11, no. 42, pp. 19658-19683, 2019.

[25] Z. Li, H. Wang, Y. Chen et al., "pH- and NIR light-responsive polymeric prodrug micelles for hyperthermia-assisted sitespecific chemotherapy to reverse drug resistance in cancer treatment," Small, vol. 12, no. 20, pp. 2731-2740, 2016.

[26] B. Yang, Y. Chen, and J. Shi, "Reactive oxygen species (ROS)based nanomedicine," Chemical Reviews, vol. 119, no. 8, pp. 4881-4985, 2019.

[27] C. Liang, L. Xu, G. Song, and Z. Liu, "Emerging nanomedicine approaches fighting tumor metastasis: animal models, metastasis-targeted drug delivery, phototherapy, and immunotherapy," Chemical Society Reviews, vol. 45, no. 22, pp. 6250-6269, 2016.

[28] M. Rinaldi, M. Caffo, L. Minutoli et al., "ROS and brain gliomas: an overview of potential and innovative therapeutic strategies," International Journal of Molecular Sciences, vol. 17, no. 6, p. 984, 2016.

[29] H. Lin, Y. Chen, and J. Shi, "Nanoparticle-triggered in situ catalytic chemical reactions for tumour-specific therapy," Chemical Society Reviews, vol. 47, no. 6, pp. 1938-1958, 2018.

[30] X. Qian, J. Zhang, Z. Gu, and Y. Chen, "Nanocatalysts-augmented Fenton chemical reaction for nanocatalytic tumor therapy," Biomaterials, vol. 211, pp. 1-13, 2019.

[31] Z. Shen, J. Song, B. C. Yung, Z. Zhou, A. Wu, and X. Chen, "Emerging strategies of cancer therapy based on ferroptosis," Advanced Materials, vol. 30, no. 12, article e1704007, 2018.

[32] M. Saeed, W. Ren, and A. Wu, "Therapeutic applications of iron oxide based nanoparticles in cancer: basic concepts and recent advances," Biomaterials Science, vol. 6, no. 4, pp. 708725, 2018.

[33] S. Wang, G. Yu, Z. Wang et al., "Enhanced antitumor efficacy by a cascade of reactive oxygen species generation and drug release," Angewandte Chemie (International Ed. in English), vol. 58, no. 41, pp. 14758-14763, 2019.

[34] G. Shim, S. Ko, D. Kim et al., "Light-switchable systems for remotely controlled drug delivery," Journal of Controlled Release, vol. 267, pp. 67-79, 2017.

[35] Z. Jia, L. Song, F. Zang et al., “Active-target T1-weighted MR imaging of tiny hepatic tumor via RGD modified ultra-small Fe3O4 nanoprobes," Theranostics, vol. 6, no. 11, pp. 17801791, 2016.

[36] X.-L. Tang, J. Wu, B.-L. Lin et al., "Near-infrared lightactivated red-emitting upconverting nanoplatform for T1weighted magnetic resonance imaging and photodynamic therapy," Acta Biomaterialia, vol. 74, pp. 360-373, 2018. 